\title{
Spectro-timing analysis of Cygnus X-1 during a fast state transition
}

\author{
M. Böck ${ }^{1}$, V. Grinberg ${ }^{1,2}$, K. Pottschmidt ${ }^{3,4}$, M. Hanke ${ }^{1}$, M. A. Nowak ${ }^{5}$, S. B. Markoff ${ }^{6}$, P. Uttley ${ }^{7}$, J. Rodriguez ${ }^{8}$, \\ G. G. Pooley ${ }^{9}$, S. Suchy ${ }^{10}$, R. E. Rothschild ${ }^{10}$, and J. Wilms ${ }^{1}$ \\ ${ }^{1}$ Dr. Karl-Remeis-Sternwarte, Astronomisches Institut der Universität Erlangen-Nürnberg, and Erlangen Centre for Astroparticle \\ Physics, Sternwartstraße 7, 96049 Bamberg, Germany \\ e-mail: Moritz.Boeck@sternwarte.uni-erlangen.de \\ 2 Universitätssternwarte, Ludwig-Maximilians Universität München, Scheinerstr. 1, 81679 Munich, Germany \\ 3 CRESST, University of Maryland Baltimore County, 1000 Hilltop Circle, Baltimore, MD 21250, USA \\ ${ }^{4}$ NASA Goddard Space Flight Center, Astrophysics Science Division, Code 661, Greenbelt, MD 20771, USA \\ 5 MIT-CXC, NE80-6077, 77 Mass. Ave., Cambridge, MA 02139, USA \\ 6 Astronomical Institute "Anton Pannekoek", University of Amsterdam, Kruislaan 403, Amsterdam, 1098 SJ, The Netherlands \\ 7 University of Southampton, Southampton SO17 1BJ, UK \\ 8 Laboratoire AIM, CEA/IRFU - Université Paris Diderot - CNRS/INSU, CEA DSM/IRFU/SAp, Centre de Saclay, \\ 91191 Gif-sur-Yvette, France \\ 9 Cavendish Laboratory, J. J. Thomson Avenue, Cambridge CB3 OHE, UK \\ ${ }_{10}$ Center for Astrophysics and Space Sciences, University of California at San Diego, La Jolla, 9500 Gilman Drive, CA 92093-0424, \\ USA
}

Received 29 April 2011 / Accepted 7 July 2011

\begin{abstract}
We present the analysis of two long, quasi-uninterrupted RXTE observations of Cygnus X-1 that span several days within a $10 \mathrm{~d}$ interval. The spectral characteristics during this observation cover the region where previous observations have shown the source to be most dynamic. Despite that the source behavior on time scales of hours and days is remarkably similar to that on year time scales. This includes a variety of spectral/temporal correlations that previously had only been observed over Cyg X-1's long-term evolution. Furthermore, we observe a full transition from a hard to a soft spectral state that occurs within less than $2.5 \mathrm{~h}-$ shorter than previously reported for any other similar Cyg X-1 transition. We describe the spectra with a phenomenological model dominated by a broken power law, and we fit the X-ray variability power spectra with a combination of a cutoff power law and Lorentzian components. The spectral and timing properties are correlated: the power spectrum Lorentzian components have an energy-dependent amplitude, and their peak frequencies increase with photon spectral index. Averaged over $3.2-10 \mathrm{~Hz}$, the time lag between the variability in the 4.5-5.7 keV and 9.5-15 keV bands increases with decreasing hardness when the variability is dominated by the Lorentzian components during the hard state. The lag is small when there is a large power law noise contribution, shortly after the transition to the soft state. Interestingly, the soft state not only shows the shortest lags, but also the longest lags when the spectrum is at its softest and faintest. We discuss our results in terms of emission models for black hole binaries.
\end{abstract}

Key words. X-rays: binaries - X-rays: individuals: Cyg X-1

\section{Introduction}

The observed behavior of most black hole (BH) X-ray binaries (XRBs) can be classified into two major states, the socalled hard, power-law dominated, and the soft, thermally dominated state (e.g., Remillard \& McClintock 2006, and references therein; these authors also introduce the most extreme steep power law state seen in only a few BHs). In the hard state the $\mathrm{X}$-ray continuum can be described by a hard power law, which is interpreted as resulting from Comptonization in a hot $\left(k T_{\mathrm{e}} \approx\right.$ $50-100 \mathrm{keV}$ ) electron plasma surrounding the accretion disk, or in a jet (Dove et al. 1998; Haardt \& Maraschi 1993; Zdziarski et al. 2002; Markoff et al. 2005). A strong contribution of jet synchrotron emission is found in some sources (Markoff et al. 2001; Russell et al. 2010; Laurent et al. 2011). In the case of Cyg X-1 the X-ray continuum can be modeled with a broken power law hardening above a break energy $E_{\text {break }} \approx 10 \mathrm{keV}$ and a highenergy cutoff with a folding energy of $\sim 100 \mathrm{keV}$ (e.g., Wilms et al. 2006). In the hard state, BH XRBs are generally observable in the radio with a flat/inverted radio through IR spectrum
(Fender et al. 2004, 2009; Dunn et al. 2010). X-ray variability above $0.1 \mathrm{~Hz}$ during this state is high (rms $>20 \%)$. In the soft state the X-ray spectrum is dominated by emission from an accretion disk and the core radio/IR emission is quenched. Cyg X-1, however, retains a strong power law component even in its softest X-ray spectra.

In most Galactic BHs, transitions between states are common. The state behavior generally can be described as part of a characteristic evolution of spectral and timing states that depends on the previous source history. Over the outburst of an $\mathrm{X}$-ray transient, the source is seen to move along a q-shaped track in a hardness intensity diagram (HID; e.g., Fender et al. 2004; Homan \& Belloni 2005). The outburst starts in the hard state; during the initial phase, the source brightens without significantly changing its spectral shape, and the radio/IR flux increases. At some point, the X-ray spectrum starts to soften. The radio/IR flux becomes more erratic. In some sources radio ejections were seen during this intermediate state (Corbel et al. 2004; Fender et al. 2004, 2006; Wilms et al. 2007). After the state transition, the source spectrum is soft and no core radio/IR emission 
is detected (Fender et al. 2009). During this soft state phase the bolometric source flux decreases, until at some lower luminosity the X-ray spectrum hardens, and the source becomes detectable in the radio/IR again. During the final part of the outburst the source is back in the hard state and the source luminosity decreases until the XRB ceases to be observable (Fender et al. 2004; Belloni et al. 2006).

The observed behavior of BH XRBs is determined by the interplay between emission from the accretion disk and from the regions of the accretion inflow/outflow in which the hard X-rays and the radio emission are generated. Transitions between the two states are therefore especially suited to place constraints on the physics of these components. Despite the fact that the outbursts can last weeks or months, the transitions between hard and soft states are for most sources rare and often fast. Belloni et al. (2006), for example, report a hard to soft transition of GX 339-4 that occurred in less than $10 \mathrm{~h}$. For this reason, the behavior during state transitions is not well studied. In addition, comparisons between different sources are complicated because the influence of other variables, such as the variation of the mass accretion rate, is difficult to ascertain. Here we will present a detailed analysis of a state transition as observed in a single object.

The canonical BH candidate Cygnus X-1 is a persistent XRB that spends most of the time in the hard state; however, it shows frequent excursions into soft states (Wilms et al. 2006, and references therein), including aborted transitions ("failed state transitions"; Pottschmidt et al. 2000, 2003). Because of its brightness and persistent emission, Cyg X-1 is one of the best studied BHs. In addition to studies of snapshot observations (e.g., Gierliński et al. 1999; Di Salvo et al. 2001; Frontera et al. 2001; Makishima et al. 2008; Hanke et al. 2009), most recent work has concentrated on the long-term behavior of the source from a monitoring campaign with the Rossi X-Ray Timing Explorer (RXTE) at a two week sampling that started in 1998 and is still ongoing (Pottschmidt et al. 2003; Gleissner et al. 2004a,b; Axelsson et al. 2005; Wilms et al. 2006, and references therein). The motivation of the present work was to fill the gap between the biweekly time scale and individual RXTE pointings with an intensive quasi-continuous observation spanning 10 days. Our aim was to determine differences and similarities between the behavior of Cyg X-1 on time scales of minutes, hours, days, and the long-term behavior found during the monitoring campaign. As we will show in the following, this observation covered an especially interesting time when a state transition occurred.

The remainder of this paper is structured as follows: in Sect. 2 we describe the observations and data analysis methods, the results of which are presented in Sects. 3 and 4. The results are discussed in Sect. 5.

\section{Observations and data analysis}

This paper is based on 78 RXTE orbits of observations of Cygnus X-1 on 2005 February 01-11 (MJD 53 402-53412; RXTE proposal ID 90127). We used data from all three instruments onboard RXTE, namely the All Sky Monitor (ASM; Levine et al. 1996), the Proportional Counter Array (PCA; Jahoda et al. 2006) and the High Energy X-ray Timing Experiment (HEXTE; Rothschild et al. 1998). We reduced data from PCA and HEXTE with HEASOFT 6.3.1 following Wilms et al. (2006), but using data from all xenon layers and making use of the improvement of background models, which now allow us to consider data taken at least 10 min after passages through the South Atlantic Anomaly (SAA; see Fürst et al. 2009). Spectra and lightcurves with a time resolution of $16 \mathrm{~s}$ were obtained us-
Table 1. Energy bands of the RXTE-PCA used in this paper according to the energy conversion of PCA Epoch 5.

\begin{tabular}{lcc}
\hline \hline PCA Channels & Energy (PCU0) & Energy (PCU1234) \\
\hline $11-13$ & $4.53-5.82 \mathrm{keV}$ & $4.49-5.71 \mathrm{keV}$ \\
$23-35$ & $9.72-15.4 \mathrm{keV}$ & $9.40-14.76 \mathrm{keV}$ \\
\hline
\end{tabular}

ing the standard2f mode PCA data. To characterize the source's spectral shape on this short time scale using hardness studies, light curves were extracted for the two channels listed in Table 1, as well as for the whole energy range of the PCA. For the timing analysis light curves with a time resolution of $\sim 2 \mathrm{~ms}\left(2^{-9} \mathrm{~s}\right)$ were extracted in the same energy channels. The calculation of Fourier-frequency dependent quantities such as power spectra, $\mathrm{X}$-ray time lags, and the coherence function follows Nowak et al. (1999a). Data modeling and all other data reduction was performed with ISIS 1.4.9 (Houck \& Denicola 2000; Noble et al. 2006; Noble \& Nowak 2008).

To study the spectral variability of the source, we extracted spectra for each RXTE orbit, yielding 78 spectra with a PCA exposure of about $3 \mathrm{ks}$ each. We considered PCA data between $2.8 \mathrm{keV}$ and $50 \mathrm{keV}$, and HEXTE data in the energy range $18-250 \mathrm{keV}$. For the fourth and fifth PCA bin $(2.8-3.2 \mathrm{keV}$ and $3.2-3.6 \mathrm{keV}$ ) we added systematic errors of $1 \%$ and $0.5 \%$, respectively ${ }^{1}$. A multiplicative constant was used to account for the different PCA and HEXTE flux calibration. The constant for the HEXTE had typical values of about $85 \%$ with respect to the PCA.

Hard X-ray spectra of BHs are traditionally explained using Comptonization, either in a Compton corona or in a jet (Wilms et al. 2006; Markoff et al. 2005, and references therein). A disadvantage of these models, however, is that the spectral parameters obtained from these models are often not unique (Nowak 2008; Nowak et al. 2011). It is still unclear which of the several models used in the literature is the correct physical interpretation of the data. Following Wilms et al. (2006), we therefore modeled the X-ray spectra using a purely empirical spectral model, an absorbed broken power law with exponential cutoff. In the following, we will denote the power law indices below and above the break energy $E_{\text {break }} \approx 10 \mathrm{keV}$ with $\Gamma_{1}$ and $\Gamma_{2}$, respectively. A Gaussian profile was added to model the Fe $\mathrm{K} \alpha$-line at $6.4 \mathrm{keV}$. For the thermal emission of the accretion disk the diskbb model (Mitsuda et al. 1984) was applied. This phenomenological model with $\Gamma_{1}>\Gamma_{2}$ describes the continuum with its spectral hardening above $10 \mathrm{keV}$ very well, with reduced $\chi^{2}$ values around 1 . We note that it is possible to map a spectrum defined through $\Gamma_{1}$ and $\Gamma_{2}$ to the more physical models, using the correlations found by Wilms et al. (2006). Clear correlations between $\Gamma_{1}$ and the other parameters of the empirical model allow us to identify the X-ray spectral state of the source with just one parameter, simplifying the analysis of the state dependence of X-ray timing and radio properties.

In addition to the X-ray data, quasi-simultaneous radio observations were made at $15 \mathrm{GHz}$ with the University of Cambridge's Ryle Telescope. The time resolution was $8 \mathrm{~s}$, using interleaved observations of a nearby phase reference source

\footnotetext{
1 Based on modeling all Cyg X-1 spectra from the years-long RXTE monitoring campaign we found this selection for the two low-energy bins to provide reliable absorption and disk parameters. We did not add the recommended systematic errors of $0.5 \%$ to the remaining PCA bins because the reduced $\chi^{2}$ of fits to hard state observations of Cyg X-1 can be significantly below 1 in this case, indicating an overestimation of the uncertainties.
} 

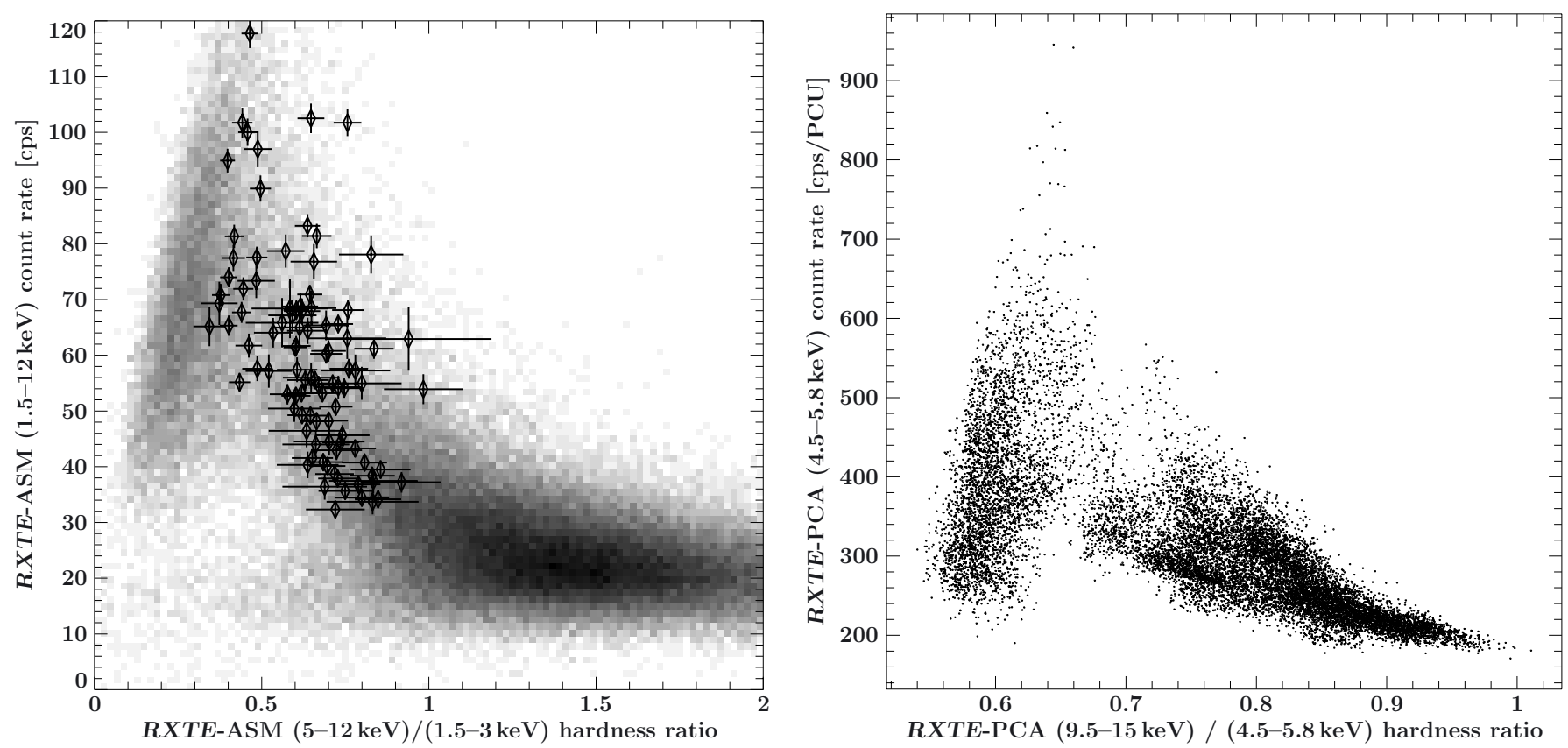

Fig. 1. Left: hardness-intensity-diagram of Cyg X-1 using RXTE-ASM data. The hardness is defined here as the ratio of the 5-12 keV and the 1.5-3 keV ASM count rates; the intensity is the total 1.5-12 keV ASM count rate (see also Fender et al. 2006, their Fig. 5). The grayscale represents the single-dwell (90 s) ASM-data from 1996 January until 2010 August. Their characteristic density forms two regions in the HID, indicating two different states. The data analyzed in this work (black symbols) cover the transition region. Right: the transitional nature of the observation is confirmed by the HID calculated from 16 s PCA data.

and observations of 3C 48 and 3C 286 as flux density calibrators. The telescope uses linearly-polarized feeds, and the measurements represent Stokes' I+Q; the flux density scale is believed to be consistent with the scale of Baars et al. (1977), after allowing for the difference in polarizations. The overall amplitude scale is believed to be accurate to about $5 \%$, and the noise level on a single 8-s sample is about $12 \mathrm{mJy}$ (the data shown in Fig. 2 have been averaged over $10 \mathrm{~min}$, and so the thermal noise is about $1.4 \mathrm{mJy}$ ).

\section{X-ray spectral variability}

\subsection{An hour-long state transition}

To characterize the evolution of the source's overall spectral properties, in Fig. 1 we show the HID of Cyg X-1 as obtained from the single-dwell $90 \mathrm{~s}$ RXTE-ASM data taken between 1996 and 2010. In this diagram, the hard state is found at a hardness of $>1$ and at count rates $\lesssim 40 \mathrm{cps}$. Transitions into the soft state are characterized in the ASM by a softening of the spectrum correlated with a brightness increase, followed by a further softening and a brightness decrease, leading to a $\Lambda$-like shape in this HID. The right panel of Fig. 1 shows a similar HID, calculated from the RXTE-PCA 16 s lightcurves during the 2005 February observation only. The figure shows that at this time resolution a $\Lambda$-shaped transitional region between hard and soft state in the HID is covered. In the following we will consider the two regions covered in the HID obtained with RXTE-PCA as a "hard" and "soft" region instead of "hard intermediate" and "soft intermediate" regions.

In order to investigate the potential occurrence of a state transition in more detail we now turn to the spectral parameters of the broken power-law modeling of our orbit-resolved PCA and HEXTE spectra. The X-ray and radio lightcurves and the evolution of the spectral parameters are shown in Fig. 2.
During the 10 days of the 2005 February observation, we find $2.0<\Gamma_{1}<2.7$. For broken power-law models, Wilms et al. (2006) show that $\Gamma_{1}>2.1$ is consistent with transitional and soft states. Thus the range of $\Gamma_{1}$-values during our observation confirms the conclusion from the HID that Cyg X-1 spent these days close to the transitional region between the hard and the soft state. A closer inspection of Fig. 2 shows that at MJD 53 409.7, from one RXTE pointing to the next, Cyg X-1 changed its photon index from $\Gamma_{1}=2.39$ to $\Gamma_{1}=2.55$ within only $2.25 \mathrm{~h}$ (Fig. 2, panel e) and moved in the HID from the harder region to the softer region. No other transition between the two regions was included in these data. The change is also seen in the more prominent disk, whose peak temperature increased to $0.25-0.40 \mathrm{keV}$ and whose inner radius moved inward to a mean value of $17 \pm 3 r_{\mathrm{G}}$ from $56 \pm 5 r_{\mathrm{G}}$ before the transition ${ }^{2}$. A drop in $N_{\mathrm{H}}$ after the transition could be caused by increased ionization of the stellar wind by the much softer X-ray spectrum. The duration of the transition was only a few hours, much shorter than the day-long transitions previously reported for Cyg X-1 (e.g., Cui et al. 1997, 2002) and comparable with the very short transition seen in GX 339-4 by Belloni et al. (2006).

\subsection{Spectral variability on time scales from hours to days}

In contrast to previous observations, which consisted either of a few hour-long pointed observations or of several ks long observations spaced by weeks, the present data set also allows us to study the spectral variability of Cyg X-1 during a transitional state. Figure 3 compares the variability of the two photon indices, $\Gamma_{1}$ and $\Gamma_{2}$, during the 2005 February observation, with that observed in the long-term monitoring between 1999 and 2004

\footnotetext{
${ }^{2}$ Values of $r_{\text {in }}$ were obtained from the diskbb normalization assuming a distance of $2 \mathrm{kpc}$, a black hole mass of $10 M_{\odot}$, and an inclination of $35^{\circ}$ (Herrero et al. 1995).
} 


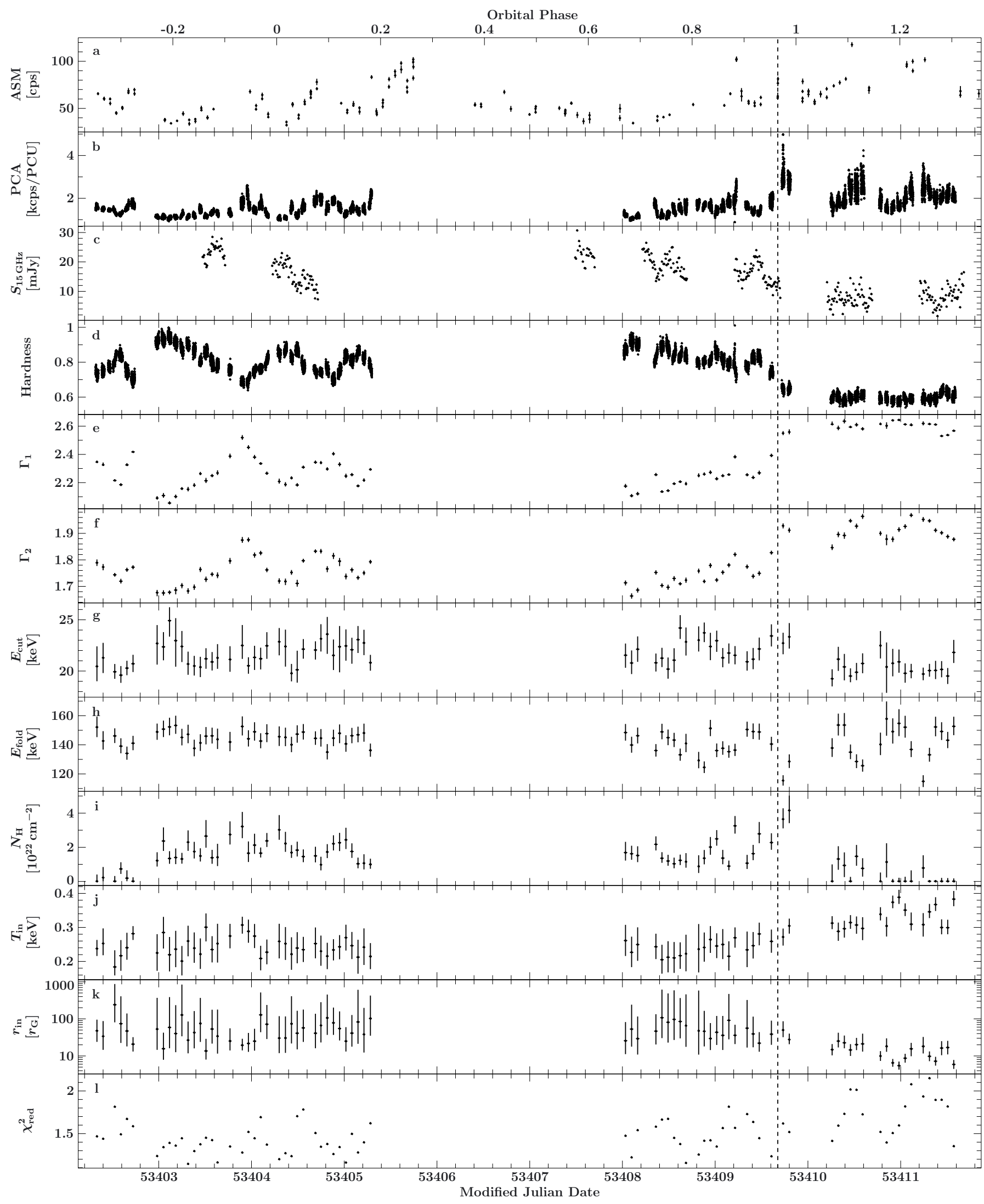

Fig. 2. Temporal evolution of the count rates and spectral parameters of Cyg X-1 during the observation period. The upper three panels are the light curves of Cyg X-1 measured with a) RXTE-ASM (1.5-12 keV); b) PCA (total energy range 2-60 keV) and c) the Ryle Telescope (at $15 \mathrm{GHz}$ ). d) The hardness is the same as in Fig. 12, namely the ratio of the PCA count rates in the energy bands $(9.5-15 \mathrm{keV}) /(4.5-5.8 \mathrm{keV})$. The bottom panels show the temporal evolution of $\mathbf{e}$ ) the photon index $\left.\Gamma_{1} ; \mathbf{f}\right)$ the photon index above the spectral break $\Gamma_{2} ; \mathbf{g}$ ) the cutoff and $\left.\mathbf{h}\right)$ folding energy of the high-energy cutoff; i) the hydrogen column; j) the inner temperature and $\mathbf{k}$ ) the inner radius of the accretion disk; and $\mathbf{l}$ ) the reduced $\chi^{2}$ of the best fits. The dashed vertical line indicates the transition. 


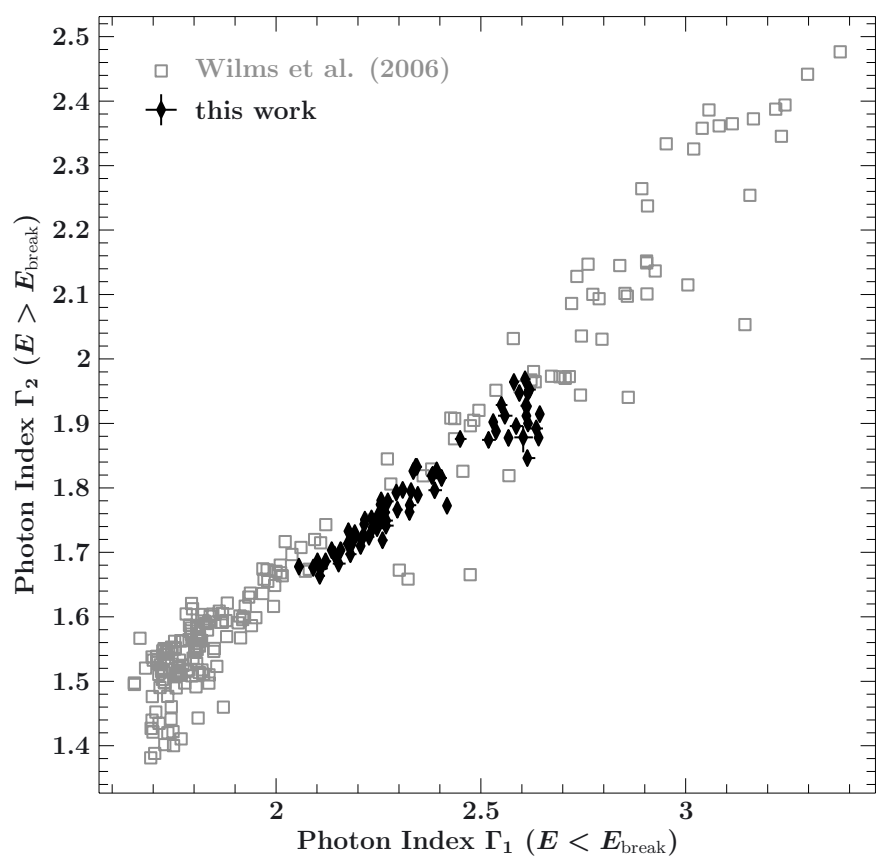

Fig. 3. Correlation between the photon indices below and above the break energy, derived here for single orbit observations, is identical to the linear correlation present in the long-term spectral evolution from 1999-2004 (Wilms et al. 2006). Note that this correlation extends across hard and soft states.

(Wilms et al. 2006). During the $10 \mathrm{~d}$ of our observation, the variation of $\Gamma_{1}$ and $\Gamma_{2}$ covered $\sim 33 \%$ of the parameter space covered by the long-term monitoring, where $1.6<\Gamma_{1}<3.4$ was found.

This general similarity between the monitoring on time scales of hours and weeks also extends to the broad-band radio to X-ray spectral variability. The relation between the RXTE orbitwise determined X-ray photon index $\Gamma_{1}$ and the simultaneously measured $15 \mathrm{GHz}$ radio flux, was consistent with the relation yielded by complete observations in the long-term monitoring campaign (Fig. 4). In the hourly and in the weekly monitoring we observe that the radio flux is seen to increase while the X-ray spectrum softens up to $\Gamma_{1} \approx 2.2$, and the radio lightcurve shows flaring behavior. Above this threshold, i.e., during the transition into the soft state, the radio flux decreases.

These results indicate that the overall spectral variability of Cyg X-1 on time scales of hours is similar to that seen on time scales of months. The large scatter seen from one monitoring observation to the next is due to the fact that the variability of the properties of the source on time scales of hours covers a significant fraction of the overall variability of the source seen in the $\mathrm{X}$-ray monitoring. Because the radio and X-ray fluxes also show similar behavior down to time scales of hours, the region where the radio and X-ray flux are produced must be small.

We expect the tight correlation between the X-rays and the radio to break down on time scales of minutes, especially during flaring episodes, which are interpreted as ejections of hot electron bubbles from the accretion flow into the jet (van der Laan 1966; Hjellming \& Johnston 1988; Gleissner et al. 2004a). Because the radio emission is produced farther downstream in the jet, a delay between the radio and X-rays is expected during these events, as was indeed seen in the one flare reported by Wilms et al. (2007) for Cyg X-1 and is also observed in other microquasars, in particular in GRS 1915+105 (Mirabel et al. 1998; Klein-Wolt et al. 2002; Prat et al. 2010), where it was interpreted as the ejection of the corona (Rodriguez et al. 2008). The most

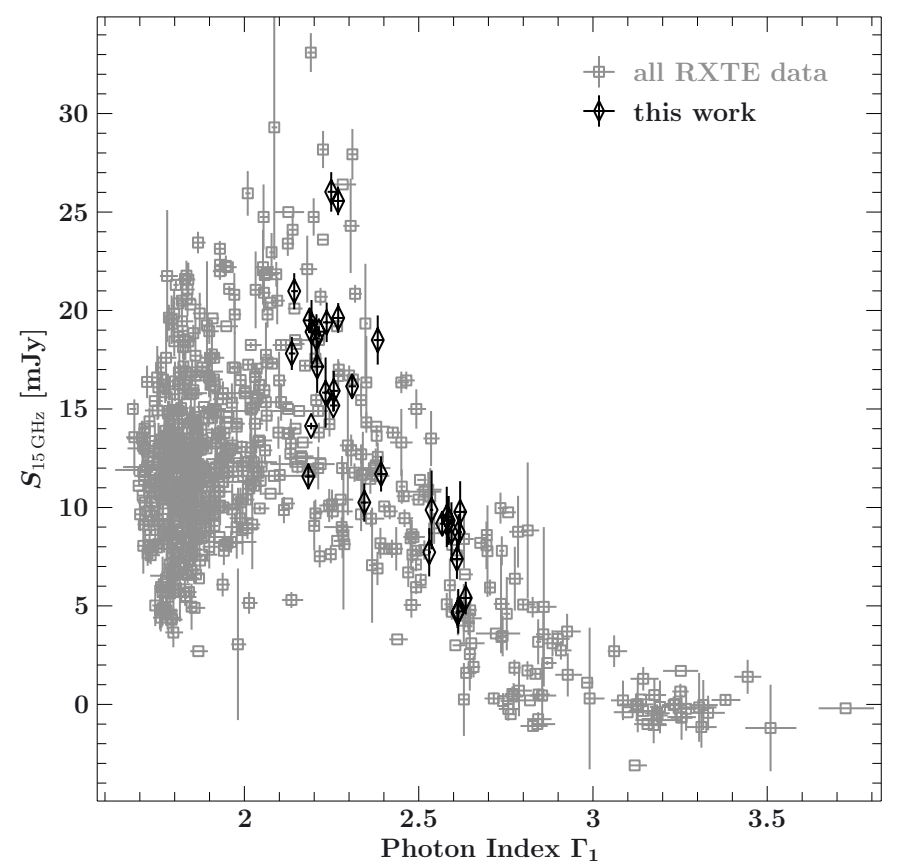

Fig. 4. Relation between the $15 \mathrm{GHz}$ radio flux and the photon index $\Gamma_{1}$.

likely flaring event during our observation occurred directly after the state transition, when the PCA light curve in its total energy range of about $2-60 \mathrm{keV}$ peaked to 5000 counts $\mathrm{PCU}^{-1}$, one of the highest measured Cyg X-1 count rates ever observed with RXTE. This count rate was reached although the absorption was high with a hydrogen column of $3.6_{-0.5}^{+0.6} \times 10^{22} \mathrm{~cm}^{-2}$. Unfortunately, however, no simultaneous radio data are available during this time.

\section{X-ray timing behavior}

Having discussed the spectral variability on time scales of ks, we now turn to the behavior of Cyg X-1 on time scales of seconds. We will first characterize this behavior through standard power spectrum analysis in several energy bands, and then discuss the variability through Fourier cross-spectral analysis techniques, namely X-ray time lags and the coherence function.

\subsection{Power spectra}

The calculation of the power spectral density (PSD) was performed following Nowak et al. (1999a) and Pottschmidt et al. (2003). Power spectra are normalized according to Miyamoto et al. (1991), i.e.

$$
\int \operatorname{PSD}(f) \mathrm{d} f=\left(\frac{\sigma}{\mu}\right)^{2}
$$

where $\mu$ is the mean value of the light curve and $\sigma$ its standard deviation. The PSDs were modeled as the sum of a power law with an exponential cutoff and two Lorentzian profiles $L_{1}$ and $L_{2}$. We parameterize $L_{i}$ by the peak frequency $v_{i}$ where $f \times L_{i}(f)$ reaches its maximum, the quality factor $Q_{i}=f_{i} / \Delta f_{i, F W H M}$, and the strength $\mathrm{rms}_{i}$, where $\mathrm{rms}_{i}^{2}=\int_{0}^{\infty} L_{i}(f) \mathrm{d} f$ corresponds to the contribution to the relative root mean square variability. Using the peak frequency as independent parameter instead of the resonance frequency

$f_{i}=2 Q_{i} v_{i}\left(1+4 Q_{i}^{2}\right)^{-1 / 2}$ 


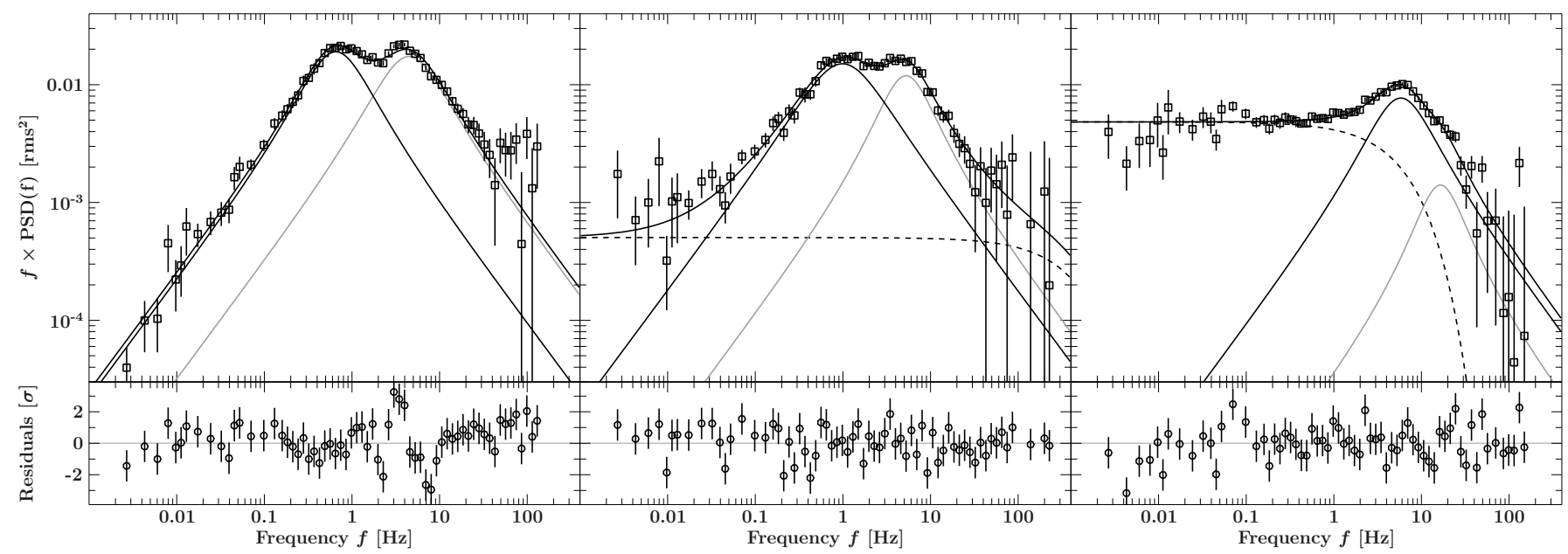

Fig. 5. Examples of characteristic power spectra. The solid gray lines show the Lorentzian profiles, and the dashed gray lines a cutoff power law Left: PSD from an observation with one of the hardest spectra in our data set. $f \times \operatorname{PSD}(f)$ vanishes for low frequencies and shows a double-humped shape, with no requirement for a power law in addition to the two Lorentzians. There is a slight indication for a third Lorentzian profile with a peak frequency between about 40-80 Hz. Middle: PSD obtained from a transitional state, requiring an additional power law at low frequencies. Right: PSD in the soft state, with yet stronger contribution of the power law. This sequence of power spectra shows that the Lorentzian peaks shift to higher frequencies as the $\mathrm{X}$-ray spectrum becomes softer.

Table 2. Correlation coefficients $\rho$ and weighted linear fits using the method of Fasano \& Vio (1988) between the Lorentzians' peak frequencies $v_{i}$ and the photon index $\Gamma_{1}$ (valid for $2.0 \leq \Gamma_{1} \leq 2.7$ ).

\begin{tabular}{lc}
\hline \hline$\rho$ & Linear correlation \\
\hline 0.98 & $\ln \left(v_{1} / \mathrm{Hz}\right)=(-8.18 \pm 0.02)+(3.70 \pm 0.01) \cdot \Gamma_{1}$ \\
0.96 & $\ln \left(v_{2} / \mathrm{Hz}\right)=(-2.03 \pm 0.01)+(1.70 \pm 0.01) \cdot \Gamma_{1}$ \\
0.98 & $\ln \left(v_{1} / \mathrm{Hz}\right)=(1.743 \pm 0.007)+(0.489 \pm 0.017) \cdot \ln \left(v_{2} / \mathrm{Hz}\right)$ \\
\hline
\end{tabular}

facilitates the fitting procedure, because $v_{i}$ is much better constrained and less dependent on $Q_{i}$ than $f_{i}$, especially for broad Lorentzians with low $Q_{i} . v_{i}$ is also the relevant parameter for correlations (e.g., Pottschmidt et al. 2003, and references therein). $L_{i}$ has thus the form

$L_{i}(f)=A_{i}\left(v_{i}^{2}-2 f_{i} f+f^{2}\right)^{-1}$,

where $f_{i}$ is defined by Eq. (2) and

$A_{i}=\operatorname{rms}_{i}^{2} v_{i}\left(1+4 Q_{i}^{2}\right)^{-1 / 2}\left[\frac{\pi}{2}+\arctan \left(2 Q_{i}\right)\right]^{-1}$.

This parameterization naturally comprises "zero frequencycentered Lorentzians" (e.g., Nowak 2000) for $Q_{i}=0$ (and thus $\left.f_{i}=0\right)$, but $v_{i}>0$.

In our earlier work based on data from 1998 to 2001, we required up to four Lorentzians to model the typical power spectrum (Pottschmidt et al. 2003). For the data analyzed here, two broad Lorentzians in addition to a power law with an exponential cutoff describe the PSDs accurately enough (see also Axelsson et al. 2005). The major reason for this difference is that the 1998-2001 data include observations with harder spectra than the data analyzed here (see also below and Fig. 5). Because a strongly rising power law with strong cutoff can affect the rms of broad Lorentzians, we froze the index of the cutoff power law to 1 . In combination with the Lorentzian profiles, this $1 / f$ "flicker noise" describes the data well, but it does not extend to the highest observed frequencies and a cutoff to the power law was required.

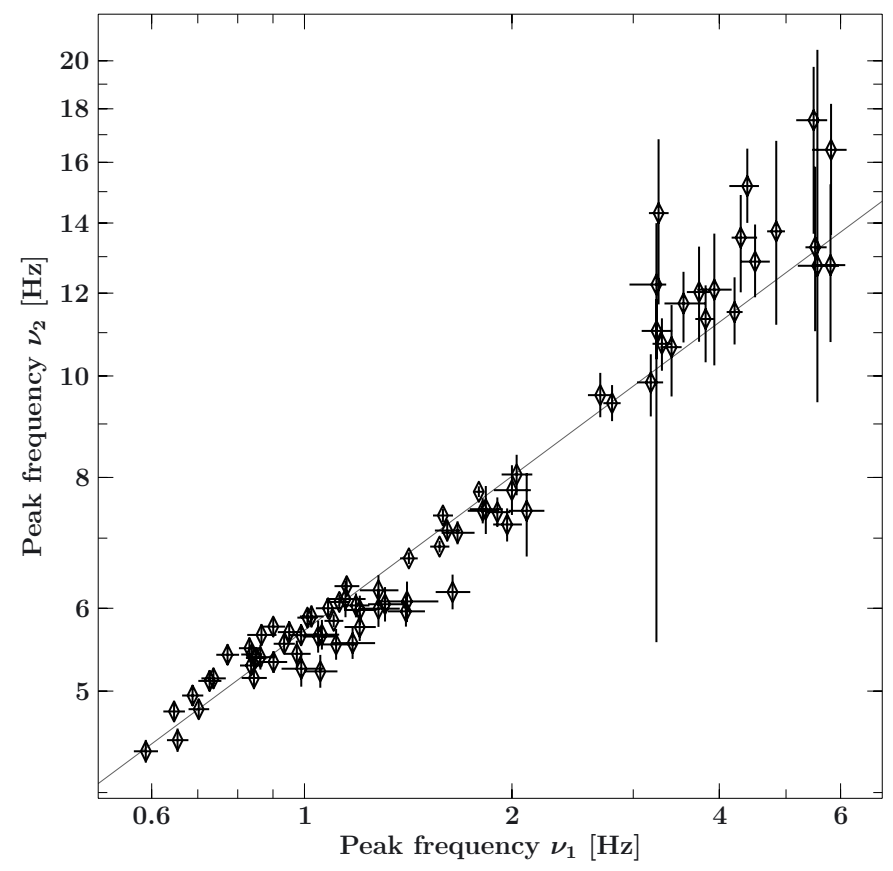

Fig. 6. Correlation between the peak frequencies $v_{1}$ and $v_{2}$ of the two Lorentzians. The gray line is the linear fit of Table 2.

\subsubsection{Correlation with the X-ray spectrum}

During the observation the peak frequency of $L_{1}, v_{1}$, varied between 0.5 and $6 \mathrm{~Hz}$, while the peak frequency of $L_{2}$ varied between 4 and $30 \mathrm{~Hz}$. These peak frequencies are clearly correlated (Fig. 6); interestingly, we find $v_{1} \propto \sqrt{v_{2}}$ (see Table 2). Figure 5 displays typical power spectra from the hardest to the softest observations. The shape of the power spectra clearly depends on the spectral state, and exhibits the following features:

- The PSDs of observations with a hard X-ray spectrum show a clear double humped profile. Individual Lorentzians can be clearly identified. A few of the hardest observations in this sample $\left(\Gamma_{1} \lesssim 2.1\right)$ show slight indications of the presence of 
M. Böck et al.: Spectro-timing analysis of Cygnus X-1 during a fast state transition

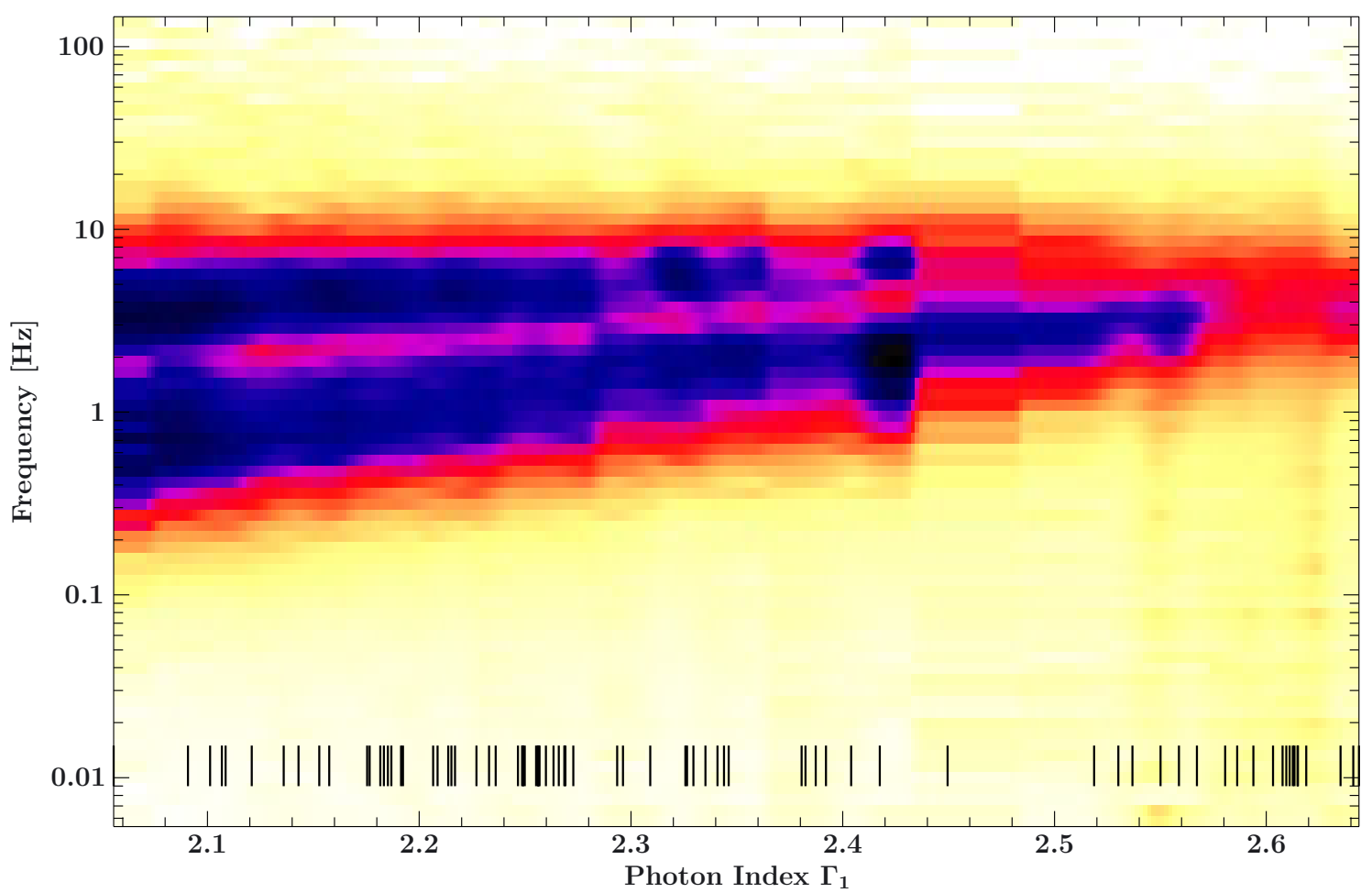

Fig. 7. Variation of the 4.5-5.8 keV power spectrum with the photon index $\Gamma_{1}$. A darker color code indicates a higher value of $f \times \operatorname{PSD}(f)$. Dashes at the bottom show the photon indices of the individual spectra. Intermediate values are interpolated with a Gaussian blur. With increasing photon index the variability components shift to higher frequencies and their intensity decreases (in this energy band $L_{2}$ completely fades out toward $\left.\Gamma_{1} \sim 2.6\right)$.

a third broad Lorentzian with a peak frequency in the range of 30-60 Hz. The contribution of the low frequencies to the variability of the corresponding light curve is very small, i.e., $f \times \operatorname{PSD}(f)$ decreases strongly toward low frequencies and no power law component is apparent.

- With softening X-ray spectrum, the low-frequency $(\$ 0.1 \mathrm{~Hz})$ variability increases. In this frequency range the PSD has a power law shape. The Lorentzian components shift to higher frequencies.

- In the spectrally softest observations the power law with exponential cutoff dominates the PSD. The rms of the second Lorentzian $L_{2}$, which is located at higher frequencies, is significantly reduced with respect to $L_{1}$.

Figure 7 shows how the power spectrum changes as a function of the photon index $\Gamma_{1}$. The image shows the humps of the PSDs as dark bands, which become weaker when the X-ray spectrum gets softer. In addition it is apparent that the peak frequencies of the Lorentzians are clearly correlated with the photon index $\Gamma_{1}$ (see also Fig. 8). See Table 2 for the numerical relation between the Lorentzian parameters and the spectral shape.

\subsubsection{Energy dependence of the variability}

Figure 9 shows the shapes of two power spectra calculated for the same observation but in different energy bands. While the qualitative shape of the PSDs is similar and the corresponding peak frequencies are found to agree within their uncertainties, the strength of the individual components of the PSD is clearly energy-dependent. The most obvious disparity is the strength of the humps. This energy dependency can be quantified using the Lorentzian model. The peak frequencies and quality factors of the individual Lorentzians are

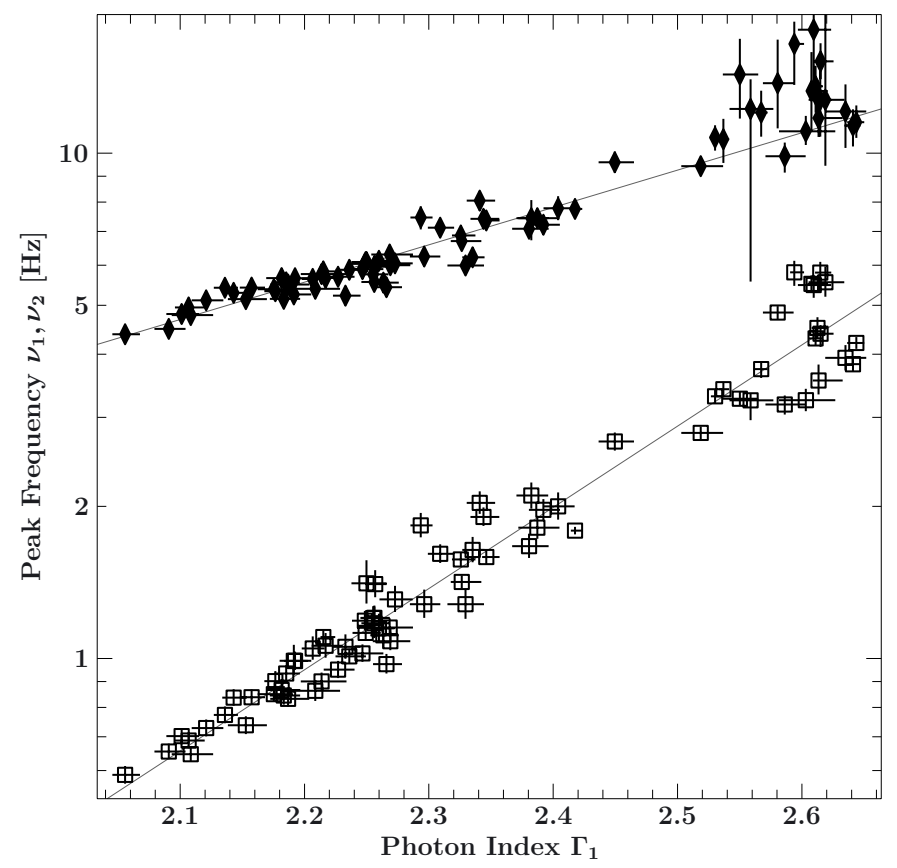

Fig. 8. Correlation between the photon index and the peak frequencies of the Lorentzian profiles. The gray lines are the linear fits of Table 2.

generally energy-band-independent within their statistical uncertainty. The rms of the Lorentzians, however, is strongly energyband-dependent.

We fitted the power spectra from both energy bands simultaneously, constraining $v_{i}$ and $Q_{i}$ to have the same value in all energy bands and leaving the Lorentzian rms a function of the 


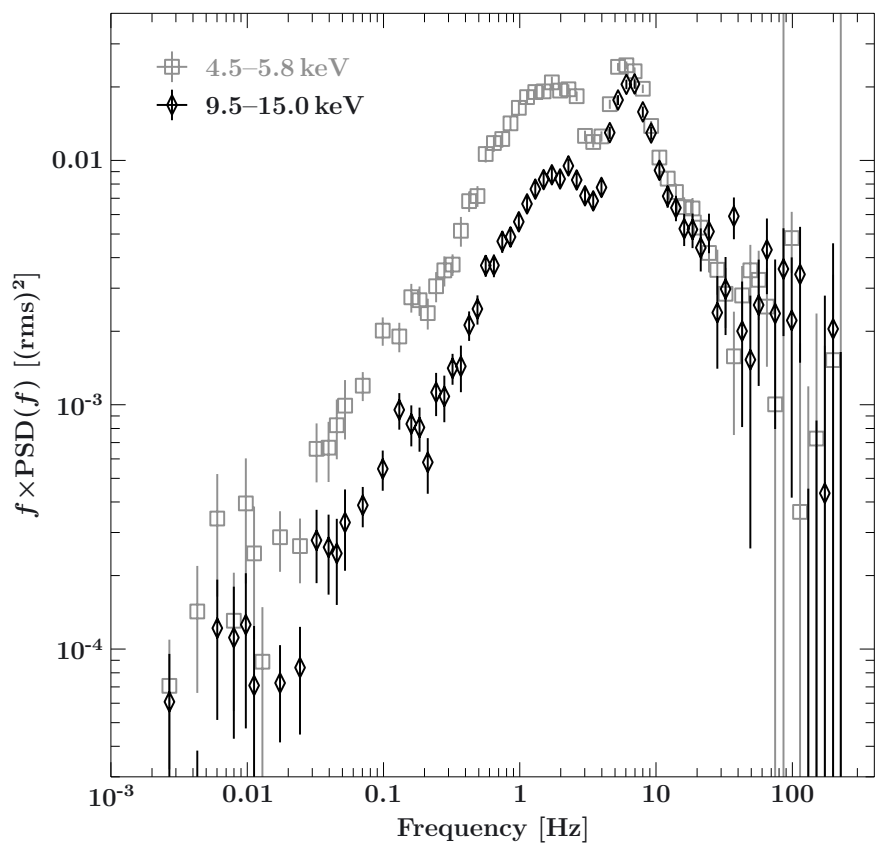

Fig. 9. PSDs of the observation 90127-01-01-05 in the low (open squares) and high (black diamonds) energy band. The photon index of this observation is $\Gamma_{1}=2.33_{-0.01}^{+0.02}$.

energy band. This approach allows us to localize the Lorentzians in the few cases when they are almost faded out in one energy band but still visible in the other one. Figure 10 shows the energy dependence of the rms for both Lorentzians.

The strength of $L_{1}$ in the $9.5-15 \mathrm{keV}$ energy band is only about two thirds of that in the $4.5-5.8 \mathrm{keV}$ band. With an rms variability of $\gtrsim 10 \%, L_{1}$ was visible in all the low-energy data analyzed here. The highest rms values for $L_{2}$ are found in the observations with the hardest X-ray spectra (see Figs. 7 and 10). At these high values, the rms for $L_{2}$ is greater in the $4.5-5.8 \mathrm{keV}$ band than in the 9.5-15 keV band. As the spectrum softens, $\mathrm{rms}_{2}$ decreases in both energy bands, but decreases more significantly in the $4.5-5.8 \mathrm{keV}$ energy band. In the softest observations, the $L_{2}$ component cannot be detected in the $4.5-5.8 \mathrm{keV}$ band, while it remains visible in the $9.5-15 \mathrm{keV}$ energy band with an rms value of a few per cent (Fig. 10, right). In the soft state a strong power law component is observed at the frequencies where the $L_{1}$ component dominates during the hard state.

\subsection{Time lags and coherence function}

While light curves at different energies are generally correlated, this relationship is frequency-dependent. The degree of linear correlation between the two light curves can be measured with the coherence function, which depends on the Fourier frequencies (a detailed discussion is given by Nowak et al. 1999a, Sect. 3.1). For two correlated time series one can define a Fourier frequency-dependent delay or time lag $\delta_{t}\left(f_{j}\right)$ (Nowak \& Vaughan 1996) as

$\delta_{t}\left(f_{j}\right)=\frac{\arg \left[S^{*}\left(f_{j}\right) H\left(f_{j}\right)\right]}{2 \pi f_{j}}=\frac{\Phi_{H\left(f_{j}\right)}-\Phi_{S\left(f_{j}\right)}}{2 \pi f_{j}}$.

Here $S\left(f_{j}\right)$ and $H\left(f_{j}\right)$ are the discrete Fourier transformations of the soft and hard X-ray lightcurves $s\left(t_{i}\right)$ and $h\left(t_{i}\right)$, and $\Phi_{S\left(f_{j}\right)}$ and $\Phi_{H\left(f_{j}\right)}$ are the corresponding phases. In Eq. (5), the phase was chosen such that a positive time lag means that the light curve in the hard energy band is delayed with respect to the soft energy band, as is common for BH XRBs. A detailed description of the calculation of time lags and coherence, including their interpretation and the calculation of their uncertainties, is given by Nowak \& Vaughan (1996).

The lag is generally a strong function of Fourier frequency. To obtain a simpler quantity, we averaged the time lag spectrum and the coherence function in the $3.2-10 \mathrm{~Hz}$ band (see Pottschmidt et al. 2003). We use these averaged coherence and time lag values in the following. Their evolution is shown in Fig. 11. In this data set the coherence was close to 1 and the time lag was usually longer than $\sim 4 \mathrm{~ms}$. After the transition to the soft state there are observations in which the time lag drops to about $2 \mathrm{~ms}$. The time lags vary strongly between about 2 and $11 \mathrm{~ms}$ after the transition, and the coherence changes rapidly. This behavior is flux-dependent: while short time lags occur at the highest count rates, the time lags are long during the soft state observations with relatively low count rates.

Figure 12 again shows the HID of the present observation. The X-ray hardness and intensity in Fig. 12 are calculated on a resolution of $16 \mathrm{~s}$, while the X-ray lags, indicated in the figure by grayscale, have been calculated on a time scale of individual RXTE orbits, which typically had an exposure of $3 \mathrm{ks}$. Different regions in the HID are dominated by different characteristic lag values. In the hard state the time lag is shortest at the largest hardness and increases as the X-ray spectrum softens. In the soft state the X-ray time lag is short at the highest count rates, while it is strongly enhanced at low count rates.

The shortest hard state lag found (about $4 \mathrm{~ms}$ ) is above the lowest values found by Pottschmidt et al. (2003), which is consistent with the fact that there are only observations with $\Gamma_{1} \gtrsim 2.0$ in our data set. Observations with harder X-ray spectra and shorter lags are not included in the sample analyzed here.

Previous work indicated that the time lags in typical soft states were quite short (2-3 ms; comparable to the shortest lags seen in hard states), and that the time lags increased during transitional states (Pottschmidt et al. 2003). The temporal evolution shown in Fig. 11 reveals that the time lags are short $(2-3 \mathrm{~ms})$ when the variability contribution of the power law noise is large, whereas the Lorentzian variabilty close to the transition is associated with long time lags (4-11 ms depending on spectral hardness). This is consistent with the results of Pottschmidt et al. (2003), where the shortest time lags in the soft state arose from variability power spectra dominated by a cutoff power law, as opposed to Lorentzian shape.

\section{Discussion and summary}

\subsection{Discussion}

\subsubsection{State transition}

The changes of the emission properties of Cyg X-1 are continuous. Because the source does not follow the complete q-shaped track in a hardness intensity diagram, which is found in outbursts of X-ray transients (Fender et al. 2004; Homan \& Belloni 2005; Dunn et al. 2010), it is difficult to specify well-defined borders between its different emission states. We find the following changes of properties: in the classical hard state of Cyg X-1 strong radio emission is observed, which is thought to originate from a steady jet. The intensity of the radio emission increases as the spectrum softens up to about $\Gamma_{1} \approx 2.1$. Above this value, when Cyg X-1 leaves its classical hard state (Wilms et al. 2006), the radio flux decreases in correlation with the hard X-ray flux (Gleissner et al. 2004a). 

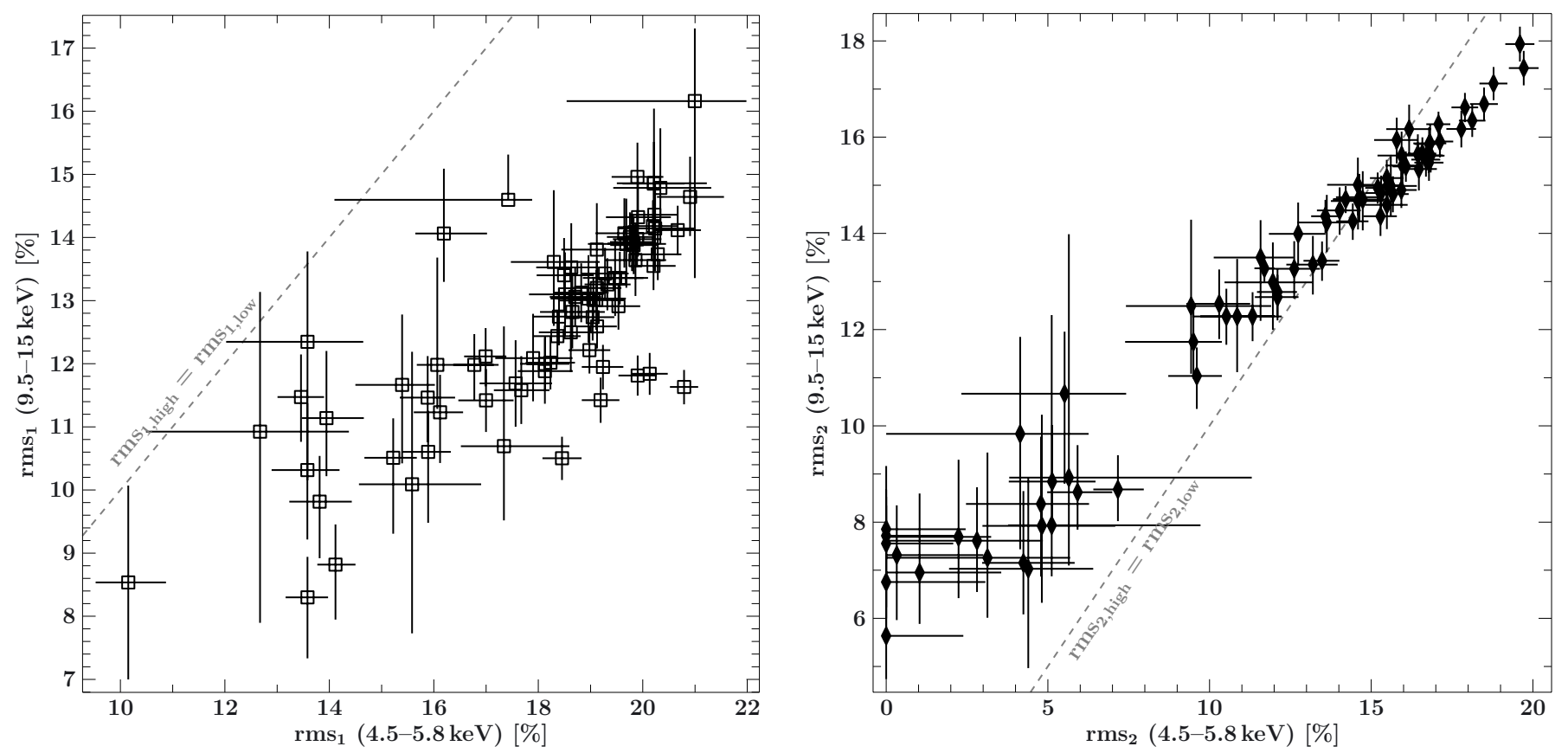

Fig. 10. Strength of the Lorentzian profiles in the two energy bands, $\mathrm{rms}_{i, \text { high }} \mathrm{vs} \mathrm{rms}_{i, \text { low }}$, for $L_{1}(l e f t)$ and $L_{2}(r i g h t)$. The dashed line is the identity.

In the transitional state data analyzed here, we see an additional change of the emission properties at $\Gamma_{1} \approx 2.5$, where the HID separates and the behavior of the time lags changes. Remarkably, this result is indicated by both spectral and timing analysis. The data points fall into two clearly separated regions in the HID, also corresponding to different power spectral shapes. Transient BH XRBs only drastically change their timing properties during transitions from "hard intermediate states" to "soft intermediate states". The fractional rms drops because of the disappearance of flat-top noise components (described as Lorentzians in this paper) in favor of a $1 / f$ (power law) noise (Miyamoto et al. 1993; Takizawa et al. 1997; Nespoli et al. 2003; Homan \& Belloni 2005; Casella et al. 2004, 2005; Belloni et al. 2005; Belloni 2009). In addition, the "soft intermediate state" power spectra may contain specific QPOs referred to as type-A and type-B (Casella et al. 2005).

Nespoli et al. (2003) found a QPO at about $6 \mathrm{~Hz}$ in an observation of GX 339-4 during a transition from the hard to the soft state. The frequency of the QPO was correlated to the PCA count rate. The power spectra on short time scales $(16 \mathrm{~s})$ of the data analyzed here did not show a comparable QPO, but it indicated that the peak frequencies of the Lorentzian profiles are correlated with the PCA count rate even on time scales shorter than one RXTE orbit. During the hard state the PCA count rate is anticorrelated with the spectral hardness. Similar to Cyg X-1, GX 339-4 shows increasing time lags as the soft count rate increases in the hard state and the flat-top noise disappears when the GX 339-4 softens into a "soft intermediate state" (Belloni et al. 2005).

\subsubsection{Noise components}

Our model consisting of only two Lorentzian profiles in addition to a power law with exponential cutoff is clearly justified by the structure of the power spectra shown in Fig. 7. A third Lorentzian is only slightly indicated in a few of our hardest observations (consistent with Pottschmidt et al. 2003), but is not required in general. Our identification of the Lorentzian profiles is equal to that of Axelsson et al. (2005, see also the discussion in their Sect. 4.1), and our results are consistent with theirs: the $v_{1}-v_{2}$ relation is identical, and it is also visible here that $\mathrm{rms}_{2}$ decreases uniformly with the peak frequency $v_{2}$.

Power spectra in the two energy bands used here can be modeled simultaneously with the normalization as the only energydependent parameter. This means that a single physical process is responsible for producing the individual variability components, but that the contribution of each process to the variability is energy-dependent.

There are different physical models describing the origin and the behavior of the noise components observed in the power spectra. Some models connect the observed frequencies with fundamental general relativistic ones of perturbed orbits near the compact object (see Nowak \& Lehr 1998, for a summary). Among others, mechanisms discussed are perturbed epicyclic frequencies, the Lense-Thirring precession frequency, and "diskoseismic" modes that are related to maximum epicyclic frequency. Our observed peak frequencies, $0.5-30 \mathrm{~Hz}$, correspond to epicyclic frequencies at about 22-345 gravitational radii for a $10 M_{\odot}$ compact object. Nowak \& Lehr (1998) point out that the "diskoseismic" frequencies are very sensitive to luminosity changes, have a low rms variability, and thus cannot be applied to systems with $\geq 10 \% \mathrm{rms}$ as in our observation. In addition, it is difficult to explain the spectral hardness of the variability components. Compared to the Lorentzian profile $L_{1}$, the one at high frequencies, $L_{2}$, is more dominant in the high-energy band, which is clearly above energies at which the disk emission contributes strongly, thus it is unlikely that both variabilities originate from the same region. Wilkinson \& Uttley (2009) observe a low-frequency Lorentzian profile originating from the accretion disk. Uttley et al. (2011) point out that the disk can produce high-frequency variations as well, which would not show up as strong soft variations, provided that they occur at small disk radii so that only a small fraction of disk flux is modulated as they propagate inward. In contrast to $L_{1}$, the absolute variability contributions of $L_{2}$, and also that of the $1 / f$ noise component, are comparable in both energy bands, however. 


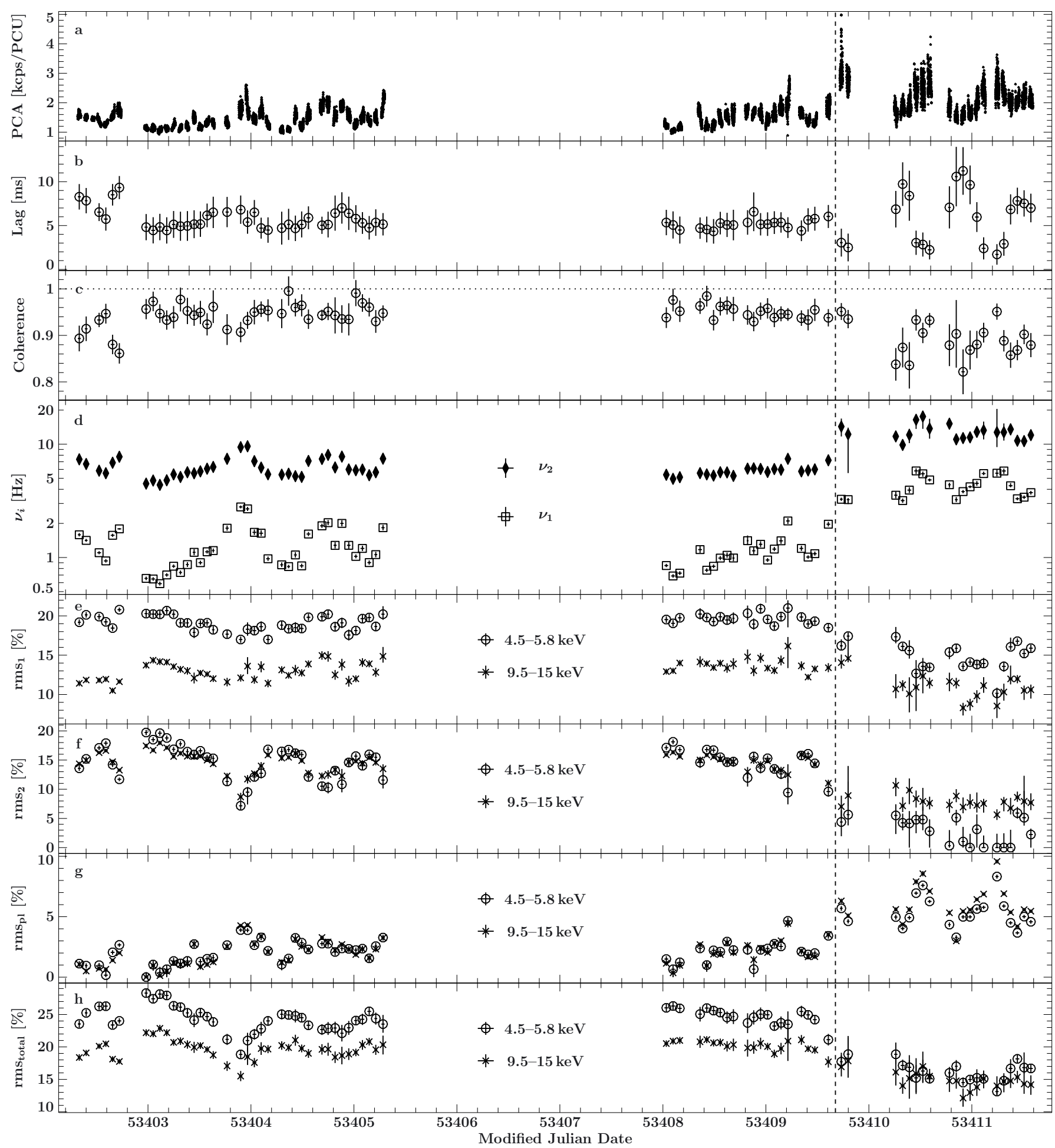

Fig. 11. Evolution of the timing parameters during the observation. The upper panel a) shows the PCA light curve of Fig. 2b for comparison; b) the averaged time lag in the $3-10 \mathrm{~Hz}$ band; c) coherence in the $3-10 \mathrm{~Hz}$ band; and d) Lorentzian peak frequencies. The following panels show the rms in both energy bands (circles for the $4.5-5.8 \mathrm{keV}$ band and crosses for the $9.5-15 \mathrm{keV}$ band) of the components: e) rms of $L_{1}$; f) of $L_{2}$; g) the power law; and h) the total rms.

Psaltis \& Norman (2000) postulate a transition radius in the inner disk acting as a low band-pass filter, which yields a dynamical model for the production of modulations, with a strong resonance to orbital, periastron- and nodal-precession. The model accounts for the observed PSDs and explains correlations between the characteristic frequencies. We find $v_{1} \propto \sqrt{v_{2}}$, which cannot be identified clearly with one of the correlations between the modes calculated by Psaltis \& Norman (2000). An extrapolation of this relation implies a characteristic frequency of $\sim 30 \mathrm{~Hz}$, at which $v_{1}=v_{2}$.

In the soft state the variability (rms) at high frequencies is lower, indicating a relatively steady state within the analyzed time scales. A possible interpretation of the power law arising in the PSDs as the spectrum softens is flicker noise in the accretion 
M. Böck et al.: Spectro-timing analysis of Cygnus X-1 during a fast state transition

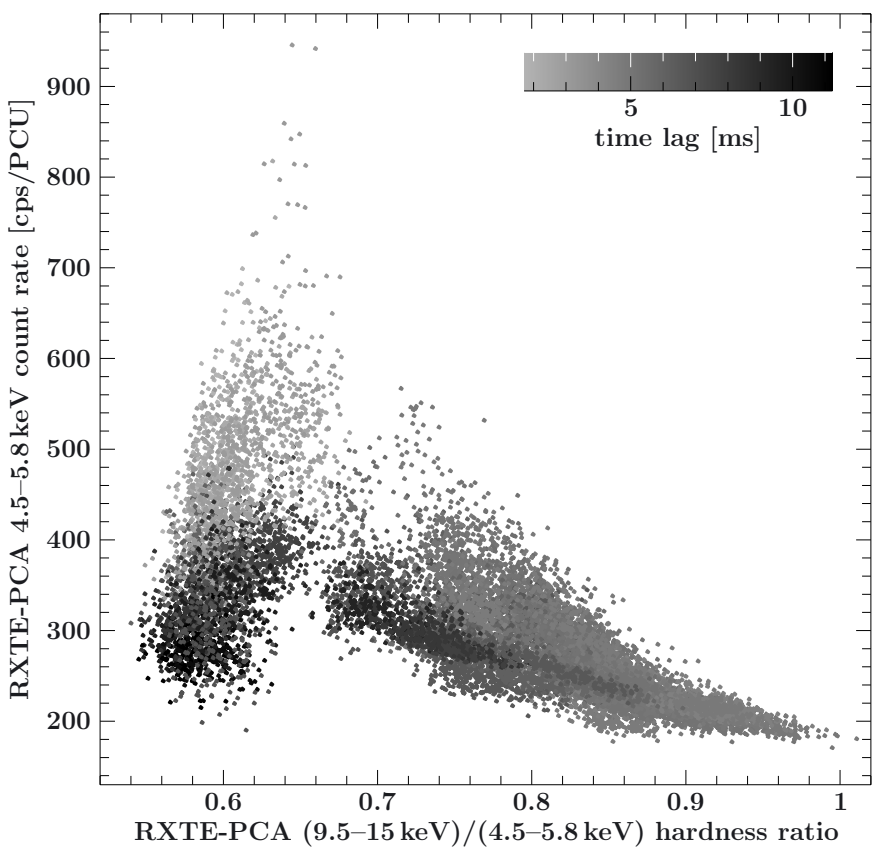

Fig. 12. X-ray lag as a function of position in the hardness intensity diagram. See text for further explanation.

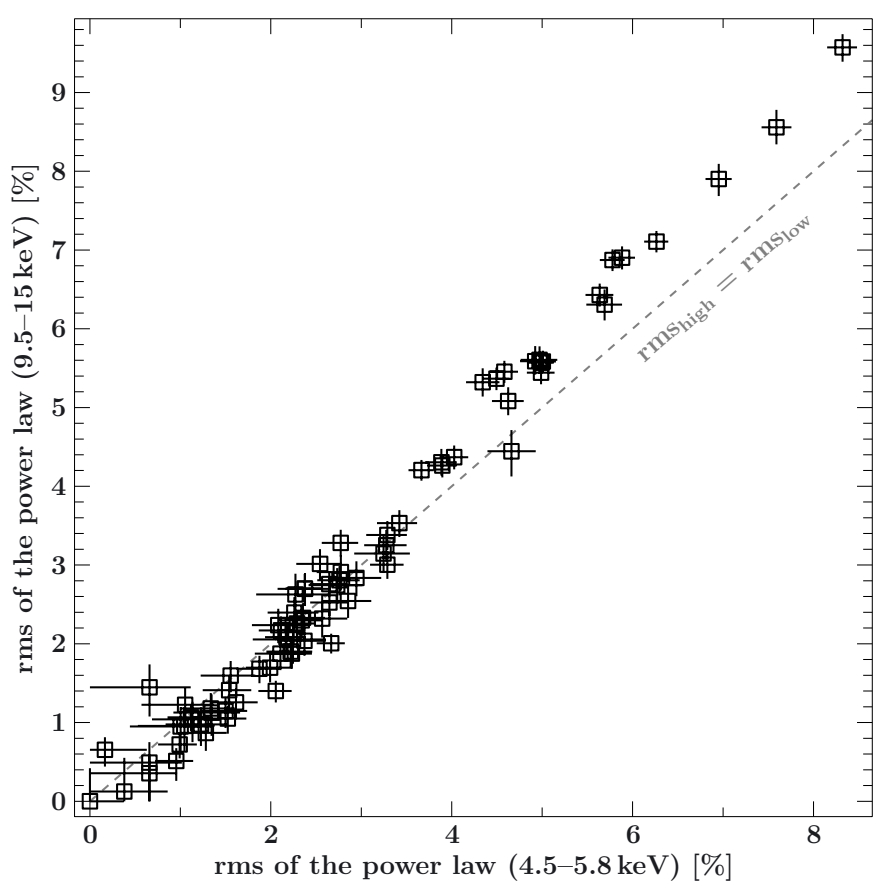

Fig. 13. Contribution of the power law to the variability in the two energy bands. The dashed line is the identity.

disk (Lyubarskii 1997). The data analyzed here indicate, however, that the variability contribution of the $1 / f$ component is strong in both energy bands and slightly stronger in the highenergy band compared to the low-energy band in the cases of strong $1 / f$ noise contribution, i.e., when the $\mathrm{X}$-ray spectrum is soft (Fig. 13). A detailed interpretation of the soft state power spectra is presented by Misra \& Zdziarski (2008), who consider linear and non-linear responses of a damped harmonic oscillator, which is assumed to govern the disk variability at each radius.

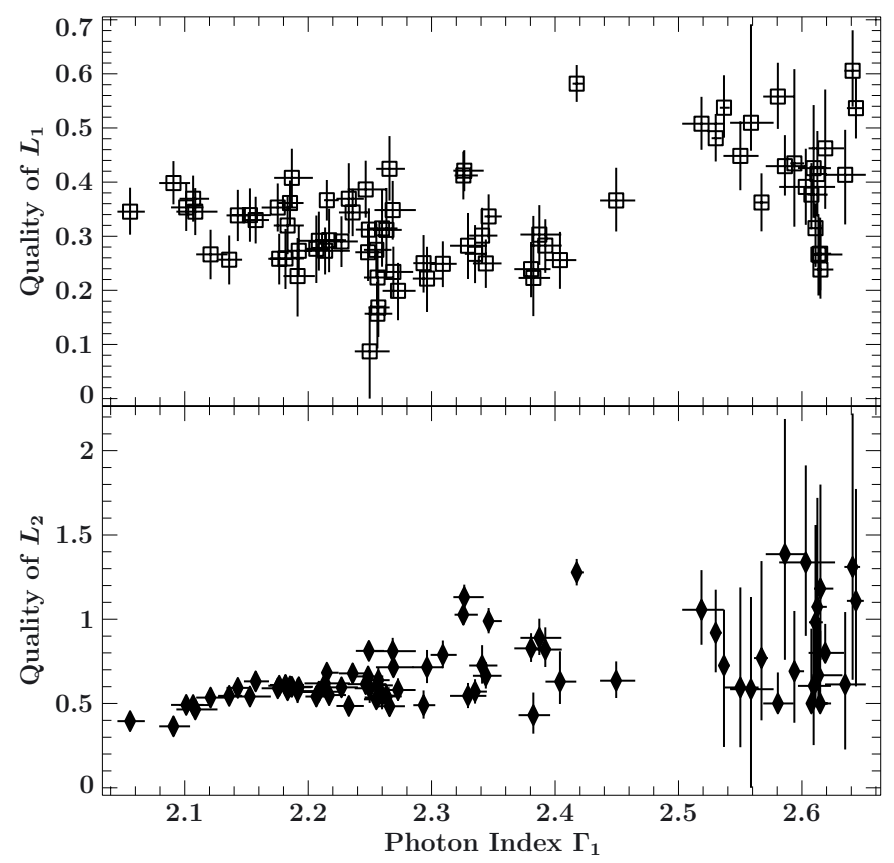

Fig. 14. The quality factor of the Lorentzian profiles.

The dependence of the Lorentzians' rms on their peak frequency (Fig. 15) is similar to that presented by Pottschmidt et al. (2003) in Fig. 6 of their work. There is, however, one slight difference: their Fig. 6 indicates a unique relation between the peak frequency and the rms of all Lorentzians below $\sim 20 \mathrm{~Hz}$, i.e., when one Lorentzian shifted to a frequency at which another Lorentzian used to be, its rms was the same as the other Lorentzian had at this frequency. This relation could be interpreted as a self-similarity, i.e., one Lorentzian profile replaces another one when it shifts to its frequency and originates from the same process or the same mode. Figure 15 does not show this behavior. Owing to the different energy dependence of the Lorentzians it is obvious that this relation depends on the considered energy channels. It is nevertheless possible that one Lorentzian replaces another one at its frequency, because when $L_{1}$ shifts to the position of $L_{2}$ other properties of the source also change. For example $v_{2}$ is about $5 \mathrm{~Hz}$ for $\Gamma_{1} \approx 2.0$, whereas $v_{1}$ reaches this frequency at $\Gamma_{1} \approx 2.7$. This change in the $\mathrm{X}$-ray spectrum implies changes in the emitting region, possibly affecting the properties of the variability components.

\subsubsection{Time lags}

Previous time lag studies of Cyg X-1 showed typically short lags around 2-3 ms in the hard state, while enhanced lags up to $9 \mathrm{~ms}$ were observed during "failed state transitions". Pottschmidt et al. (2003) used the energy bands $2-4 \mathrm{keV}$ and $8-13 \mathrm{keV}$ to obtain these values. When the source entered deeper into the soft state, the time lag returned to short values (Pottschmidt et al. 2000).

Our new data set with higher temporal resolution (RXTE orbit-wise extractions) allows us to extend these results (Figs. 11, 12). In the hard state the time lag increases with softening X-ray spectrum consistent with previous observations, whereas after the transition to the soft state the time lag behavior changes. Depending on the brightness, the time lag changes from values of up to $11 \mathrm{~ms}$ at low count rates to values of 2-3 ms at high count rates without appreciable softening of the source. Changes from long to short time lags occur from one RXTE orbit 

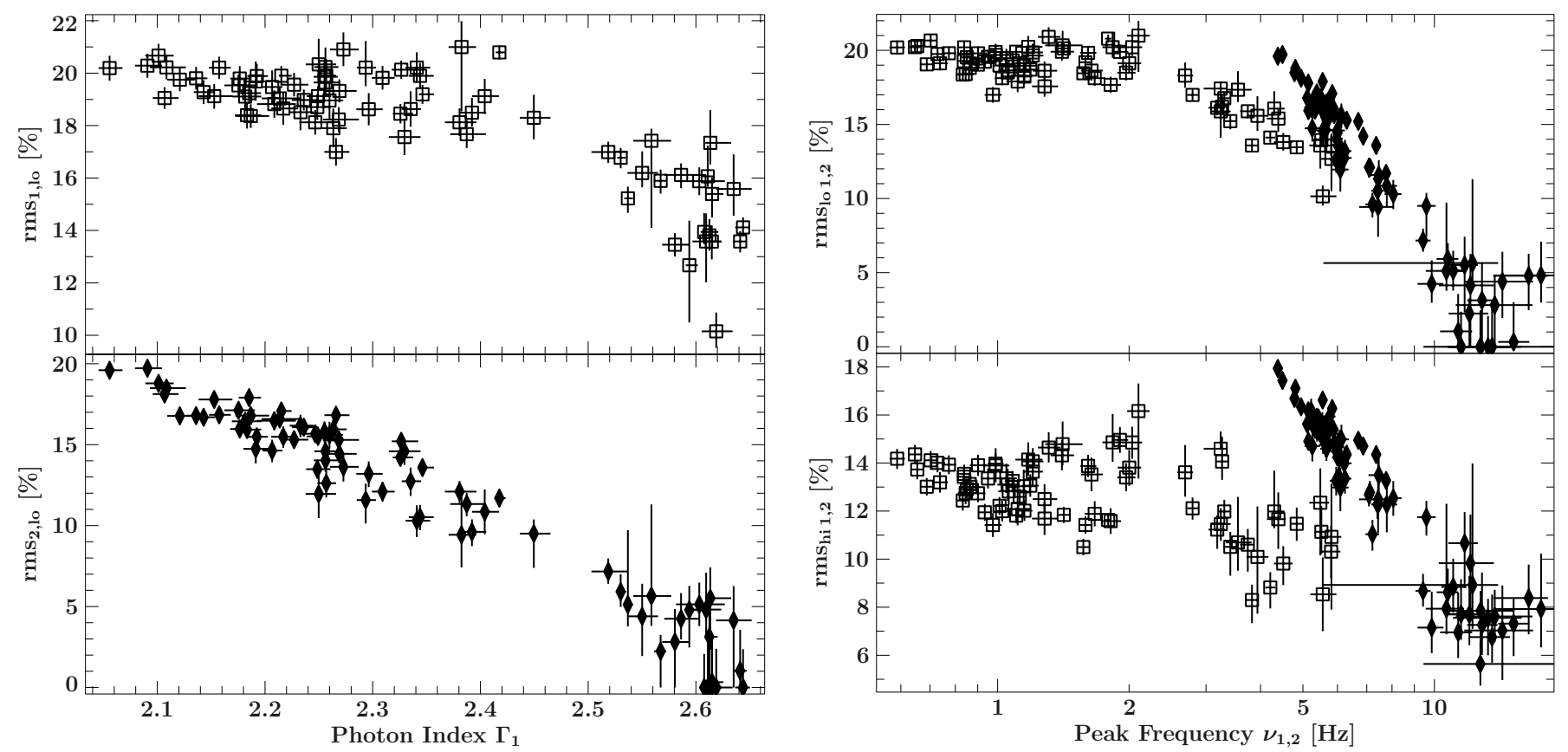

Fig. 15. Dependence of the rms of the Lorentzian profiles on the photon index (left) and on the peak frequencies (right). The open squares correspond to the first Lorentzian and the filled diamonds to the second one.

to the next. Figure 11 shows that the lag is short when the variability contribution of the power law is large, whereas the time lags of the Lorentzian variability are long depending on their peak frequency and thus on the spectral hardness.

Time lags can originate from reprocessing of radiation and propagation in the emitting region. Nowak et al. (1999b) exclude disk-intrinsic time delays as the sole reason for the lag, and discuss constraints on properties of the corona if the time lag originates in it. Kylafis et al. (2008) present a jet model that can reproduce the behavior of the time lags in the Cyg X-1 hard state. Körding \& Falcke (2004) show that time lags can be caused by a pivoting power law spectrum arising, e.g., from a jet/synchrotron model. In their work the sign of the time lag depends on the hardness/flux correlation (Körding \& Falcke 2004, Fig. 9). Interestingly, the correlation visible in the lambdashaped HIDs seems to change its sign coinciding with the change in time lag behavior, exactly at the transition: in the hard state the count rate increases with decreasing hardness, while it is the other way around in the SIMS. The transition is also where the behavior of the time lag changes.

Uttley et al. (2011) show that the time lags seen at soft X-rays $(0.5-9 \mathrm{keV})$ are caused by the accretion disk. They point out that Comptonization models for lags have difficulties, whereas propagation delays (see, e.g., Kotov et al. 2001; Arévalo \& Uttley 2006) can explain their data, and that a similar mechanism can cause time lags at higher energies.

The clear correlations between spectral and timing properties put strong constraints on models explaining the emission of accreting black holes.

\subsection{Summary}

The main results of this paper can be summarized as follows:

- CygX-1 was in an intermediate/soft state during 2005 February 01-11. The photon index $\Gamma_{1}$ reached values between 2.0 and 2.7 and was correlated with $\Gamma_{2}$ in the same way as found by Wilms et al. (2006).
- The source was very variable during that period of time; the total PCA count rate varied between 1000 and 5000 counts $\mathrm{s}^{-1}$ per PCU.

- A full transition from the hard state to the soft state occurred in less than $2.5 \mathrm{~h}$. The two states can be clearly distinguished in a hardness intensity diagram.

- Spectral and timing parameters are strongly correlated. The peak frequencies of the Lorentzian profiles shift to higher values with increasing $\Gamma_{1}$. Those are identical in the lowand the high-energy band, whereas their rms depends on the energy.

- In the HIMS the time lag increases with decreasing hardness. After the transition to the SIMS the time lag behaves differently. At high count rates $\left(\gtrsim 2500\right.$ counts $\mathrm{s}^{-1} \mathrm{PCU}^{-1}$ in this case) the time lag drops to about $2 \mathrm{~ms}$ and the contribution of the power law noise is large. At lower count rates the time lag is long, with values up to $11 \mathrm{~ms}$.

Acknowledgements. We acknowledge funding from the Bundesministerium für Wirtschaft und Technologie through the Deutsches Zentrum für Luftund Raumfahrt under contract 50OR0701. M.A.N. was supported by NASA Grant SV3-73016. J.R. acknowledges partial funding from the European Community's Seventh Framework Programme (FP7/2007-2013) under grant agreement number ITN 215212 "Black Hole Universe". We thank the International Space Science Institute, Berne, Switzerland for supporting team 116 and the X-ray group at the Center for Astrophysics and Space Sciences of the University of California at San Diego for their hospitality during the initial stages of this project.

\section{References}

Arévalo, P., \& Uttley, P. 2006, MNRAS, 367, 801

Axelsson, M., Borgonovo, L., \& Larsson, S. 2005, A\&A, 438, 999

Baars, J. W. M., Genzel, R., Pauliny-Toth, I. I. K., \& Witzel, A. 1977, A\&A, 61, 99

Belloni, T., Homan, J., Casella, P., et al. 2005, A\&A, 440, 207

Belloni, T., Parolin, I., Del Santo, M., et al. 2006, MNRAS, 367, 1113

Corbel, S., Fender, R. P., Tomsick, J. A., Tzioumis, A. K., \& Tingay, S. 2004, ApJ, 617, 1272

Cui, W., Heindl, W. A., Rothschild, R. E., et al. 1997, ApJ, 474, L57 
Cui, W., Feng, Y.X., \& Ertmer, M. 2002, ApJ, 564, L77

Di Salvo, T., Done, C., Życki, P. T., Burderi, L., \& Robba, N. R. 2001, ApJ, 547, 1024

Dove, J. B., Wilms, J., Nowak, M. A., Vaughan, B. A., \& Begelman, M. C. 1998, MNRAS, 298, 729

Dunn, R. J. H., Fender, R. P., Körding, E. G., Belloni, T., \& Cabanac, C. 2010, MNRAS, 403, 61

Fasano, G., \& Vio, R. 1988, Bulletin d'Information du Centre de Données Stellaires, 35, 191

Fender, R. P., Belloni, T. M., \& Gallo, E. 2004, MNRAS, 355, 1105

Fender, R. P., Stirling, A. M., Spencer, R. E., et al. 2006, MNRAS, 369, 603

Fender, R. P., Homan, J., \& Belloni, T. M. 2009, MNRAS, 396, 1370

Frontera, F., Palazzi, E., Zdziarski, A. A., et al. 2001, ApJ, 546, 1027

Fürst, F., Wilms, J., Rothschild, R. E., et al. 2009, Earth and Planetary Science Letters, 281, 125

Gierliński, M., Zdziarski, A. A., Poutanen, J., et al. 1999, MNRAS, 309, 496

Gleissner, T., Wilms, J., Pooley, G. G., et al. 2004a, A\&A, 425, 1061

Gleissner, T., Wilms, J., Pottschmidt, K., et al. 2004b, A\&A, 414, 1041

Haardt, F., \& Maraschi, L. 1993, ApJ, 413, 507

Hanke, M., Wilms, J., Nowak, M. A., et al. 2009, ApJ, 690, 330

Herrero, A., Kudritzki, R. P., Gabler, R., Vilchez, J. M., \& Gabler, A. 1995, A\&A, 297, 556

Hjellming, R. M., \& Johnston, K. J. 1988, ApJ, 328, 600

Homan, J., \& Belloni, T. 2005, Ap\&SS, 300, 107

Houck, J. C., \& Denicola, L. A. 2000, in Astronomical Data Analysis Software and Systems IX, ed. N. Manset, C. Veillet, \& D. Crabtree, ASP Conf. Ser., 216, 591

Jahoda, K., Markwardt, C. B., Radeva, Y., et al. 2006, ApJS, 163, 401

Klein-Wolt, M., Fender, R. P., Pooley, G. G., et al. 2002, MNRAS, 331, 745

Körding, E., \& Falcke, H. 2004, A\&A, 414, 795

Kotov, O., Churazov, E., \& Gilfanov, M. 2001, MNRAS, 327, 799

Kylafis, N. D., Papadakis, I. E., Reig, P., Giannios, D., \& Pooley, G. G. 2008, A\&A, 489, 481

Laurent, P., Rodriguez, J., Wilms, J., et al. 2011, Science, 332, 438

Levine, A. M., Bradt, H., Cui, W., et al. 1996, ApJ, 469, L33

Lyubarskii, Y. E. 1997, MNRAS, 292, 679

Makishima, K., Takahashi, H., Yamada, S., et al. 2008, PASJ, 60, 585

Markoff, S., Falcke, H., \& Fender, R. 2001, A\&A, 372, L25
Markoff, S., Nowak, M. A., \& Wilms, J. 2005, ApJ, 635, 1203

Mirabel, I. F., Dhawan, V., Chaty, S., et al. 1998, A\&A, 330, L9

Misra, R., \& Zdziarski, A. A. 2008, MNRAS, 387, 915

Mitsuda, K., Inoue, H., Koyama, K., et al. 1984, PASJ, 36, 741

Miyamoto, S., Kimura, K., Kitamoto, S., Dotani, T., \& Ebisawa, K. 1991, ApJ, 383,784

Nespoli, E., Belloni, T., Homan, J., et al. 2003, A\&A, 412, 235

Noble, M. S., \& Nowak, M. A. 2008, PASP, 120, 821

Noble, M. S., Houck, J. C., Davis, J. E., Young, A., \& Nowak, M. 2006, in Astronomical Data Analysis Software and Systems XV, ed. C. Gabriel, C. Arviset, D. Ponz, \& S. Enrique, ASP Conf. Ser., 351, 481

Nowak, M. A. 2000, MNRAS, 318, 361

Nowak, M. A. 2008, PoS, MQW7, 030

Nowak, M. A., \& Lehr, D. E. 1998, in Theory of Black Hole Accretion Discs (CUP), midsummer Symp. on Non-Linear Phenomena in Accretion Disks around Black Holes: Theory, 233

Nowak, M. A., \& Vaughan, B. A. 1996, MNRAS, 280, 227

Nowak, M. A., Vaughan, B. A., Wilms, J., Dove, J. B., \& Begelman, M. C. 1999a, ApJ, 510, 874

Nowak, M. A., Wilms, J., Vaughan, B. A., Dove, J. B., \& Begelman, M. C. 1999b, ApJ, 515, 726

Nowak, M. A., Hanke, M., Trowbridge, S. N., et al. 2011, ApJ, 728, 13

Pottschmidt, K., Wilms, J., Nowak, M. A., et al. 2000, A\&A, 357, L17

Pottschmidt, K., Wilms, J., Nowak, M. A., et al. 2003, A\&A, 407, 1039

Prat, L., Rodriguez, J., \& Pooley, G. G. 2010, ApJ, 717, 1222

Psaltis, D., \& Norman, C. 2000, unpublished [arXiv:astro-ph/0001391]

Remillard, R. A., \& McClintock, J. E. 2006, ARA\&A, 44, 49

Rodriguez, J., Hannikainen, D. C., Shaw, S. E., et al. 2008, ApJ, 675, 1436

Rothschild, R. E., Blanco, P. R., Gruber, D. E., et al. 1998, ApJ, 496, 538

Russell, D. M., Maitra, D., Dunn, R. J. H., \& Markoff, S. 2010, MNRAS, 405, 1759

Uttley, P., Wilkinson, T., Cassatella, P., et al. 2011, MNRAS, 414, L60

van der Laan, H. 1966, Nature, 211, 1131

Wilkinson, T., \& Uttley, P. 2009, MNRAS, 397, 666

Wilms, J., Nowak, M. A., Pottschmidt, K., Pooley, G. G., \& Fritz, S. 2006, A\&A, 447, 245

Wilms, J., Pottschmidt, K., Pooley, G. G., et al. 2007, ApJ, 663, L97

Zdziarski, A. A., Poutanen, J., Paciesas, W. S., \& Wen, L. 2002, ApJ, 578, 357 